\title{
Safety and Efficacy of Left Atrial Appendage Closure with the Amplatzer Cardiac Plug in Very High Stroke and Bleeding Risk Patients with Non-Valvular Atrial Fibrillation
}

Julia Kebernik · John Jose · Mohamed Abdel-Wahab · Björn Stöcker •

Volker Geist · Gert Richardt

To view enhanced content go to www.cardiologytherapy-open.com

Received: September 29, 2015 / Published online: November 13, 2015

(C) The Author(s) 2015. This article is published with open access at Springerlink.com

\section{ABSTRACT}

Introduction: Limited data exist on the outcomes after left atrial appendage closure (LAAC) with the Amplatzer ${ }^{\mathrm{TM}}$ Cardiac Plug (ACP; St. Jude Medical, Minneapolis, MN, USA) in patients with atrial fibrillation (AF) with very high stroke and bleeding risks, the subset expected to benefit most from this procedure. The objective of this study was to report clinical outcomes after LAAC with the ACP device in a very high stroke and bleeding risk cohort of patients with non-valvular AF and contraindications to oral anticoagulation (OAC).

Methods: LAAC using the ACP device was performed in 96 patients with AF who had median $\mathrm{CHA}_{2} \mathrm{DS}_{2}$-VASc and HAS-BLED scores of 5 and 3 , respectively. Post-procedure, patients

Electronic supplementary material The online version of this article (doi:10.1007/s40119-015-0053-z) contains supplementary material, which is available to authorized users.

J. Kebernik $(\varangle) \cdot$ J. Jose · M. Abdel-Wahab .

B. Stöcker · V. Geist · G. Richardt

Heart Center, Segeberger Kliniken GmbH (Academic

Teaching Hospital of the Universities of Kiel,

Lübeck, and Hamburg), Bad Segeberg, Germany

e-mail: jkebernik@gmx.de received dual antiplatelet therapy for 6 months. A transesophageal echocardiography (TEE) was scheduled at 6 months.

Results: Procedural success was $100 \%$. Procedural-related complications occurred in 7.3\% (pericardial effusion, 4.2\%; thromboembolic events, 2.1\%; device embolization, 1.0\%). Additional thromboembolic events occurred in three patients during follow-up (92.7\% follow-up). After 93.4 patient-years of follow-up, the annual rates of thromboembolic and major bleeding events were $3.2 \%$ and $1.1 \%$, respectively. In those with TEE follow-up (70\%), complete LAAC with no leaks was observed. Thrombus formation on the device was noted on TEE in two patients.

Conclusion: LAAC using the ACP device was associated with an acceptable low rate of embolic and bleeding events after a median follow-up of 9 months in a cohort of patients with AF who were amongst the highest stroke and bleeding risks reported so far in LAAC trials.

Keywords: Amplatzer ${ }^{\mathrm{TM}}$ Cardiac Plug; Atrial fibrillation; Left atrial appendage closure; Oral anticoagulation; Stroke; Thromboembolism 


\section{INTRODUCTION}

Embolic stroke is a serious complication in patients with atrial fibrillation (AF) [1]. Oral anticoagulation (OAC) is effective in reducing rates of thromboembolism, albeit with an increased bleeding risk $[2,3]$. To identify patients with a significant risk of stroke and bleeding different risk stratification scores have been developed. Whereas the $\mathrm{CHA}_{2} \mathrm{DS}_{2}$-VASc score is recommended to evaluate the individual stroke risk in patients with $\mathrm{AF}$, the HAS-BLED score was developed to assess the risk of bleeding during anticoagulation treatment [4]. Clinical trials have established the predictive value of these scores [5]. In day-to-day clinical practice, patients with the highest risk of stroke are those with a previous stroke, and patients with the highest risk of bleeding are those with a previous bleeding episode, especially the elderly. As risk factors for stroke and bleeding commonly overlap, management of patients with $\mathrm{AF}$ is still a clinical challenge and many patients at highest risk for stroke are not treated with OAC, even after the introduction of direct thrombin and factor Xa inhibitors.

New interventional therapies have been developed for stroke prevention in non-valvular AF. As about $90 \%$ of the thrombi originate from left atrial appendage (LAA) [6], LAA closure (LAAC) devices were developed to prevent thromboembolic events and to avoid long-term OAC.

Of these LAAC devices, the Watchman ${ }^{\mathrm{TM}}$ device (Boston Scientific, Marlborough, MA, USA) is the one that has been well studied. A recent meta-analysis has provided supportive data for the efficacy and safety of intervention with this device in patients with AF [7]. However, the observed effects were not uniformly in favor of the Watchman device: When compared with warfarin therapy at 2-3 years' follow-up, LAAC was associated with a decreased likelihood of hemorrhagic stroke, cardiovascular death, and non-procedural bleeding, while the rates of ischemic stroke were higher [7]. The most commonly used device in Europe is the Amplatzer $^{\mathrm{TM}}$ Cardiac Plug (ACP; St. Jude Medical, Minneapolis, MN, USA). A few studies that have been published with the ACP indicate a safe implantation with low rates of procedural-related complications [8-11]. Recent reports also suggest a reduction of stroke risk when compared to the expected stroke rate based on the $\mathrm{CHA}_{2} \mathrm{DS}_{2}$-VASc score [9-11].

Theoretically, the benefit of LAAC therapy should be more pronounced in patients with higher stroke and bleeding risks. The aim of the current analysis was to assess safety and efficacy of LAAC with the ACP device in a cohort of very high stroke and bleeding risk patients with contraindications for OAC.

\section{METHODS}

This was a retrospective observational cohort analysis of a single German center database of LAAC with the ACP device. Patients with non-valvular-AF, a $\mathrm{CHA}_{2} \mathrm{DS}_{2}$-VASc score $\geq 2$, and contraindications to OAC therapy including previous major bleeding, thromboembolic events while on OAC, high tendency to fall, intolerance and non-compliance to $\mathrm{OAC}$, and labile international normalized ratio (INR) were included.

The study cohort comprised 96 consecutive patients, who underwent LAAC with the ACP between March 2009 and December 2014. 


\section{Procedure}

Prior to the procedure, transthoracic and transesophageal echocardiography (TTE and TEE, respectively) were performed to exclude intracardiac thrombi, determine LAA anatomy, and record baseline parameters.

The procedure was performed under mild sedation (intravenous propofol and midazolam) with angiographic and TEE guidance. Access to the left atrium (LA) was gained by transseptal puncture using a Brockenbrough needle (Cook Medical, Bloomington, IN, USA) and a transseptal sheath (Cook Medical). Septum was imaged by TEE in a bicaval and a short axis view, and an inferior and posterior site was chosen for optimal LAA access. After puncture, heparin was administered at a dose of $70-100 \mathrm{U} / \mathrm{kg}$ to achieve an activated clotting time of at least $250 \mathrm{~s}$. The sheath was then introduced into LAA and angiography performed by manual contrast injection. LAA measurements were obtained by TEE and angiography (Fig. 1a). In different projections, diameters of LAA ostium, landing zone, and length were taken. Sizing was based on the dimensions of the landing zone. The device was oversized by about $4 \mathrm{~mm}$ with respect to the LAA landing zone.

Then, the ACP device was introduced and placed in the LAA using a dedicated delivery system, the Amplatzer TorqVue ${ }^{\mathrm{TM}} 45^{\circ} \times 45^{\circ}$ delivery sheath (AGA Medical Corp., Plymouth, MN, USA). Before releasing the device, stability was assessed (Minnesota wiggle maneuver, TEE, and angiographic evaluations; Fig. 1b, c). Assessments were made for any pericardial effusion during the procedure.

In all cases, intravenous ciprofloxacin (400 mg) was administered during the procedure. Dual antiplatelet therapy (DAPT) consisting of aspirin (100 $\mathrm{mg})$ and clopidogrel (75 mg) were given for at least 6 months unless there was a contraindication. After 6 months, single antiplatelet therapy was recommended lifelong. Postprocedural TTE evaluation was done to rule out pericardial effusion and to confirm proper device position.

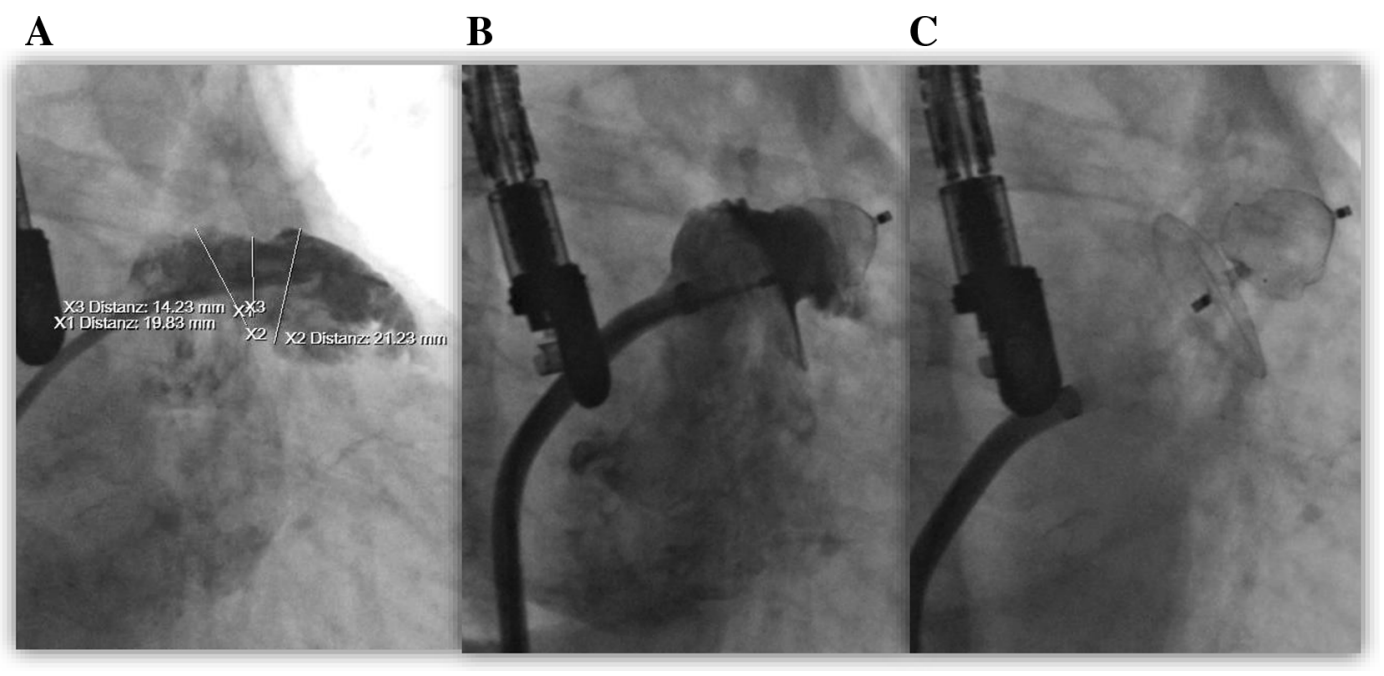

Fig. 1 Key steps of ACP implantation. a Angiographic measurement of the landing zone. $\mathbf{b}$ Angiography confirmation of an optimal position with no residual leak. c Post-release cine image frame of the device 


\section{Follow-up}

Clinical follow-up was carried out during hospital visit or through phone contact at 6 months and 1 year. Patients were scheduled a TEE at 6 months follow-up to assess the presence of a thrombus and a residual leak (mild $<1 \mathrm{~mm}$, moderate $1-3 \mathrm{~mm}$, severe $>3 \mathrm{~mm})$.

\section{Endpoints}

Procedural success was defined as successful implantation of the ACP in LAA with no residual leak by angiographic and echocardiographic evaluation.

Complications that occurred during the procedure/hospitalization and during the clinical follow-up were documented. They were defined as: thromboembolic event (transient ischemic attack (TIA), stroke, and systemic embolism), major bleeding (Bleeding Academic Research Consortium [BARC] type 23b) [12], device embolization, myocardial infarction, and all-cause mortality.

\section{Statistical Methods}

Continuous variables were expressed as mean \pm standard deviation and as median (interquartile range) depending on the data and its distribution. Categorical variables were reported as counts (percentages). Efficacy of the device to prevent thromboembolic events was tested by comparing the actual event rate at follow-up with the $\mathrm{CHA}_{2} \mathrm{DS}_{2}$-VASc score $[4,5]$ estimated event rates. The average annual risk for the whole study population was calculated. The total number of thromboembolic events during follow-up period was divided by the total patient-years of follow-up and was multiplied by 100 to get the actual annual rate of thromboembolism. Risk reduction of thromboembolism was calculated as follows: (estimated percent event rate-actual percent event rate)/estimated percent event rate.

Bleeding risk reduction was assessed analogous to stroke risk reduction. The annual event rate at follow-up was compared with the HAS-BLED score [13, 14] estimated event rates. Comparisons between observed and predicted thromboembolic and bleeding event rates were assessed using binomial tests. Rate ratios with 95\% Poisson exact confidence intervals of observed and expected rates were also calculated. STATA $^{\circledR}$ version 14 (StataCorp LP, College Station, TX, USA) and GraphPad Prism ${ }^{\circledR}$ 6 (GraphPad Software, Inc., La Jolla, CA, USA) were used for the statistical analysis.

\section{Compliance with Ethics Guidelines}

All procedures followed were in accordance with ethical standards of the responsible committee on human experimentation (institutional and national) and with the Helsinki Declaration of 1964, as revised in 2013. Informed written consent for the procedure was obtained from all patients.

\section{RESULTS}

A total of 96 consecutive patients were included in this study. Patient's baseline characteristics are summarized in Table 1 . Mean age of the study population was $76 \pm 7$ years and $39.5 \%$ were female. AF was permanent in 52\%, persistent in 25\%, and paroxysmal in the remainder. Sixty-three patients $(65.5 \%)$ had a previous stroke and 59 patients $(61.4 \%)$ had a history of major bleeding. One-fifth of the patients had a concomitant history of stroke and bleeding. The median (interquartile range) $\mathrm{CHA}_{2} \mathrm{DS}_{2}$-VASc score was 5 (4.25-7) and the 
Table 1 Baseline characteristics of the study population $(n=96)$

\begin{tabular}{|c|c|}
\hline Characteristic & Value \\
\hline Age, years, mean $\pm S D$ & $76 \pm 7$ \\
\hline Female & $38(39.5)$ \\
\hline \multicolumn{2}{|l|}{ Atrial fibrillation type } \\
\hline Paroxysmal & $22(23)$ \\
\hline Persistent & $24(25)$ \\
\hline Permanent & $50(52)$ \\
\hline $\begin{array}{l}\mathrm{CHA}_{2} \mathrm{DS}_{2} \text {-VASc score, median } \\
\text { (interquartile range) }\end{array}$ & $\begin{array}{l}5.0 \\
(4.25-7)\end{array}$ \\
\hline Congestive heart failure & $19(19.7)$ \\
\hline Hypertension & $93(96.8)$ \\
\hline Diabetes mellitus & $30(31.2)$ \\
\hline Previous stroke & $63(65.5)$ \\
\hline Vascular disease & $64(66.6)$ \\
\hline $\mathrm{CHADS}_{2}$ score, median (interquartile range) & $4(3-4.75)$ \\
\hline $\begin{array}{l}\text { HAS-BLED score, median (interquartile } \\
\text { range) }\end{array}$ & $3(3-4)$ \\
\hline History of bleeding & $59(61.4)$ \\
\hline Intracranial & $35(59.1)$ \\
\hline Gastrointestinal & $17(28.7)$ \\
\hline $\begin{array}{l}\text { Other (urethral, ophthalmological, severe } \\
\text { hematoma) }\end{array}$ & $7(11.8)$ \\
\hline Thromboembolic events on OAC & $16(16.6)$ \\
\hline High tendency to fall & $12(12.5)$ \\
\hline Intolerance and non-compliance to $\mathrm{OAC}$ & $6(6.2)$ \\
\hline Labile INR & $3(3.1)$ \\
\hline
\end{tabular}

Data expressed as number (\%) unless otherwise stated $O A C$ oral anticoagulants, INR International normalized ratio, $S D$ standard deviation

median (interquartile range) HAS-BLED score was 3 (3-4). A history of major bleeding was the principal contraindication for OAC and consequent LAAC. Other indications for LAAC were high tendency to fall $(n=12,12.5 \%)$, and intolerance as well as non-compliance for OAC
( $n=6,6.2 \%)$. Notably in $16(16.6 \%)$ patients, LAAC was performed because of thromboembolic events while on OAC.

\section{Procedural Outcome}

Successful device implantation was achieved in all patients $(n=96,100 \%)$. The mean procedural time was $46 \pm 5 \mathrm{~min}$. The most commonly used device size was $24 \mathrm{~mm}$ $(n=34,35.5 \%)$. LAAC was possible with the initial chosen device in 91 patients (95\%). In five patients, device release criteria were not met and another device size was chosen (smaller sized device in four cases). LAAC was not combined with other procedures.

Procedural-related complications occurred in seven patients (7.3\%) and are listed in Table 2. Pericardial effusion requiring pericardiocentesis was the most common complication and occurred in four patients $(4.2 \%)$. None of the patients required surgical intervention. Device embolization occurred in one patient; the device embolized into the LA (Fig. 2), where it was snared out, reintroduced and successfully implanted.

There were two cases with a thromboembolic event (2.1\%): One TIA (this was associated with device embolization) and one stroke that was attributed to hypotension. There were no procedural-related myocardial infarctions or deaths.

\section{Follow-up Outcomes}

Clinical follow-up was available in 89 patients $(92.7 \%)$ with a median (interquartile range) follow-up duration of 9 months (6-18 months). Eighteen patients (20\%) were followed up for more than 20 months, and for 35 patients (39.3\%) at least 1-year follow-up was available. 
After 93.4 patient-years of follow-up, a total of 13 events (14.6\%) were recorded (Table 3 ). Three of them were thromboembolic events: One TIA and two strokes. One TIA occurred after 18 months in an 89-year-old male patient who had a previous ischemic stroke $\left(\mathrm{CHA}_{2} \mathrm{DS}_{2}\right.$-VASc score $\left.=6\right)$ as well as an intracranial bleeding event (HAS-BLED score $=5$ ). TEE did not reveal thrombus on the device.

In a 76-year-old female patient with previous stroke $\left(\mathrm{CHA}_{2} \mathrm{DS}_{2}\right.$-VASc score $\left.=5\right)$, cranial computed tomography showed a sub-acute posterior inferior cerebellar arterial (PICA) and superior cerebellar artery (SCA) infarction on the left side 20 months after ACP implantation. This patient had no TEE follow-up. Cardioembolic stroke was the clinical diagnosis made by neurologists in both these cases.

Another patient, a 75-year-old with previous history of stroke $\left(\mathrm{CHA}_{2} \mathrm{DS}_{2}\right.$-VASc score $\left.=5\right)$, had a recurrent stroke 14 months after ACP

Table 2 Procedure related complications (total patients, $n=96)$

\begin{tabular}{ll}
\hline Complication & $\boldsymbol{n}(\%)$ \\
\hline Total & $7(7.3)$ \\
Major bleeding & $4(4.2)$ \\
Pericardial effusion & $4(4.2)$ \\
Other major bleeding & $0(0.0)$ \\
Thromboembolic events & $2(2.1)$ \\
Stroke & $1(1)$ \\
Transient ischemic attack & $1(1)$ \\
Systemic embolization & $0(0.0)$ \\
Device embolization & $1(1.0)$ \\
Myocardial infarction & $0(0.0)$ \\
Death & $0(0.0)$ \\
\hline
\end{tabular}

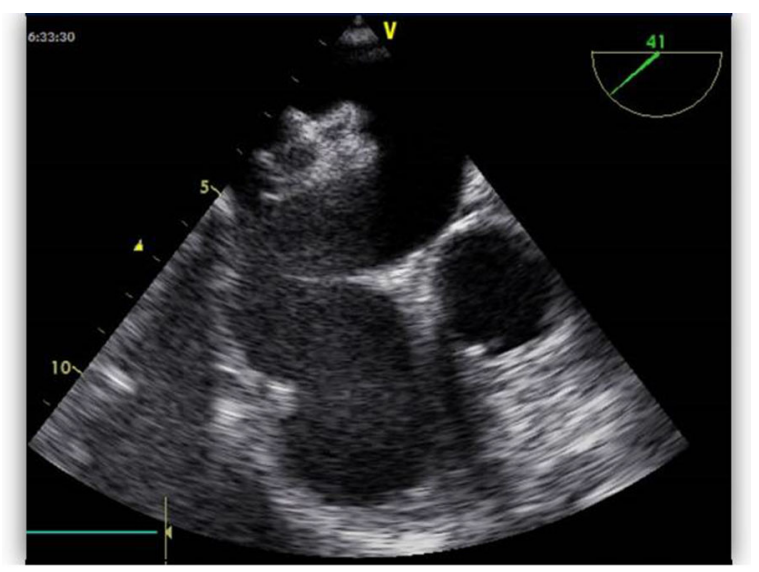

Fig. 2 Device embolization. Intraprocedural transesophageal echocardiography: embolization of the device into the left atrium

implantation while on aspirin. Magnetic resonance imaging confirmed an acute right-sided thalamic infarct and showed microangiopathic disease. TEE did not reveal thrombus on the device in this patient as well.

Major bleeding occurred in one patient at follow-up. An 82-year-old male with a HAS-BLED score of 5 experienced an intracranial bleeding, caused by a fall 26 months after device implantation, while on aspirin. One patient experienced a clinically significant pericardial effusion 11 months after the procedure. A total of nine patients died during follow-up (Table 3).

\section{TEE Outcomes}

Sixty-two patients (70\%) with clinical follow-up had a TEE evaluation after a mean duration of 8.6 months. None of the patients had a residual leak. Thrombus formation on the device was observed in two cases (3.2\%), 5 and 6 months after LAAC. Both of them were on DAPT. The presence of thrombus did not correlate with a clinical event. In both of the patients, thrombus resolved after 5 and 6 weeks of OAC therapy. 
Table 3 Follow-up outcome (total patients with follow-up, $n=89$ )

\begin{tabular}{lc}
\hline Outcome & $\boldsymbol{n}(\%)$ \\
\hline Total & $13(14.6)$ \\
Thromboembolic events & $3(3.4)$ \\
Stroke & $2(2.2)$ \\
Transient ischemic attack & $1(1.1)$ \\
Systemic embolism & $0(0.0)$ \\
Major bleeding & $1(1.1)$ \\
Intracranial bleeding & $1(1.1)$ \\
Myocardial infarction & $0(0.0)$ \\
Device embolization & $0(0.0)$ \\
Death & $9(10.1)$ \\
Cardiovascular cause & $1(1.1)$ \\
Other & $3(3.4)$ \\
Unknown & $5(5.6)$ \\
\hline
\end{tabular}

\section{DISCUSSION}

The prevention of thromboembolism is the most important therapeutic goal in patients with AF. Vitamin $\mathrm{K}$ antagonists (VKA) effectively reduce strokes rates [2], but as individual stroke and bleeding risks increase in parallel, physicians face a therapeutic dilemma concerning their use in very high stroke and bleeding risk patients.

One effective alternative to VKA are the novel oral anticoagulants (NOACs), i.e., dabigatran, rivaroxaban, apixaban, and edoxaban [15-17], whose main toted benefit in comparison to VKA is reduction of intracranial bleeding. But major bleedings still occur with these agents, especially in the elderly with renal impairment, and so far specific antidotes such as idarucizumab [18] are still in their investigational stage or undergoing phase three trials. In this context, the non-pharmacological treatment option of LAAC—which offers simultaneous advantages of stroke reduction as well as avoidance of long-term $\mathrm{OAC}$-gains prominence.

The PLAATO System (ev3, Plymouth, MN, USA) was the first dedicated LAAC device [19]. This device was succeeded by the Watchman device, whose safety and efficacy were proved in two large randomized trials. The PROTECT AF (ClinicalTrials.gov identifier, NCT00129545) [20] and the PREVAIL (ClinicalTrials.gov identifier, NCT01182441) [21] trial were non-inferiority trials that evaluated the Watchman device compared with warfarin in patients with AF who were eligible to take OAC. As stroke prevention strategies are particularly challenging in patients who are ineligible to take OAC, the ASAP study (ClinicalTrials.gov identifier, NCT00851578) [22] was designed. This small, non-randomized trial documented a reasonable safety profile over short-term follow-up for the Watchman device.

Current available data with the ACP are derived from several small, observational studies [8-11]. In contrast to the Watchman trials, most of the patients enrolled in these studies were not suitable for long-term OAC and were treated with DAPT post-implantation. The largest conducted study so far on the ACP device was recently published in 2015 by Tzikas et al. [11], which was a multicenter trial of 1047 patients that established its safety and efficacy.

Because LAAC makes long-term OAC unnecessary, patients with very high stroke and bleeding risks should benefit most from this therapy. Nevertheless, LAAC carries inherent hazards especially in the periprocedural period and therefore a net clinical benefit of LAAC has to be established even in this particular subset of patients. 
Our study population represents a very high-risk cohort of patients with AF with respect to stroke and bleeding, given the high median $\mathrm{CHA}_{2} \mathrm{DS}_{2}$-VASc score and HAS-BLED score of $5 \quad($ mean $=5.6)$ and $3 \quad($ mean $=3)$, respectively. The frequency of a previous stroke and bleeding episode were as high as $65.5 \%$ and $61.4 \%$, respectively; $20 \%$ had a concomitant history of both events. Comparing stroke and bleeding risk with previous published studies, this study cohort is amongst the highest stroke and bleeding risks reported so far. In comparison, the mean $\mathrm{CHA}_{2} \mathrm{DS}_{2}$-VASc score of the study population in the PROTECT AF trial was $3.5 ; 18.5 \%$ had a previous stroke [7]. In the largest study reported so far with the ACP device, the mean $\mathrm{CHA}_{2} \mathrm{DS}_{2}$-VASc score was 4.4 with a history of previous stroke in 39\% [11].

\section{Procedural Outcomes}

Our study confirms the results of previous studies: LAAC with the ACP has a high rate of procedural success and an acceptable rate of periprocedural complications. In recently reported studies, periprocedural complications were similar to those observed in our study, namely pericardial effusion, thromboembolic events, and device embolization. Postulated reasons for pericardial effusion are transseptal puncture related, extensive manipulation within the LAA, device recapture and repositioning, stiff wire exchange in the LAA, and extensive oversizing of the device [23, 24]. Pericardiocentesis alone seems to be sufficient enough to control this complication, its occurrence should decrease with more technical experience.

Device embolization is a well-known complication of LAAC with an average reported rate of less than $4 \%$ [25]. In our study, the device was successfully captured by a gooseneck snare. Our experience is in agreement with previous published cases of device embolization, which show that device embolizations into the LA can be successfully managed percutaneously. The reason for embolization in our case is unclear since this occurred despite ensuring a stable and safe device position. After retrieval, the same device was reintroduced and reimplanted successfully. Nonetheless, device embolization is a serious complication and more knowledge regarding its mechanisms is necessary.

\section{Device Efficacy}

Thromboembolic events during follow-up were observed at an annual rate of 3.2\%. Comparing this observed rate with the estimated annual risk of $6.7-10.0 \%$ for patients without warfarin and with a $\mathrm{CHA}_{2} \mathrm{DS}_{2}$-VASc score of 5, a 52-68\% reduction was calculated (Fig. 3). Rate ratio for incidence of thromboembolic events was 0.32 (95\% CI 0.07-0.94), assuming estimated annual risk to be $10 \%$. Reduction of thromboembolic events with ACP device in other case series varies from $59 \%$ to $80 \%$ [9-11]. Variabilities in risk reduction seem to be influenced by clinical characteristics of the patients including the $\mathrm{CHA}_{2} \mathrm{DS}_{2}$-VASc score. It must also be considered that when comparing risk reduction rates across different studies, the values of the expected stroke rate for a given $\mathrm{CHA}_{2} \mathrm{DS}_{2}$-VASc score used in the calculation of the relative risk reduction were not the same. Our results have confirmed that the reduction of thromboembolic events with the ACP device is significant even in very high stroke risk patients with $\mathrm{AF}$, the derived magnitude of the benefit being large.

During the follow-up period, one patient had a fall-related intracranial bleeding and was 
classified as major bleeding event, but in reality was an event unrelated to the device. Apart from this patient, there were no overt bleeding events recorded during follow-up. Comparing an annual rate of $1.1 \%$ bleeding episodes during follow-up in our study with the estimated annual bleeding risk of $3.74-5.8 \%$ for patients on warfarin with a HAS-BLED score of 3 , a $70-81 \%$ reduction of the bleeding risk was calculated (Fig. 3). Rate ratio for incidence of bleeding events was 0.18 (95\% CI 0.00-1.03), assuming estimated annual risk to be $5.8 \%$.

Of interest in our study was the presence of device-related thrombus, which was not associated with any thromboembolic events. The two patients who had thrombi on the device were on DAPT at the time of diagnosis (mean 5.5 months post-implant). Device-related thrombus was reported in other LAAC studies as well, although association with clinical stroke was rare. In the PROTECT AF study [25], device-associated thrombi were observed in $4.2 \%$, and thrombus-associated annualized stroke rate was $0.3 \%$. Interestingly, the rate of device-related thrombus in PROTECT

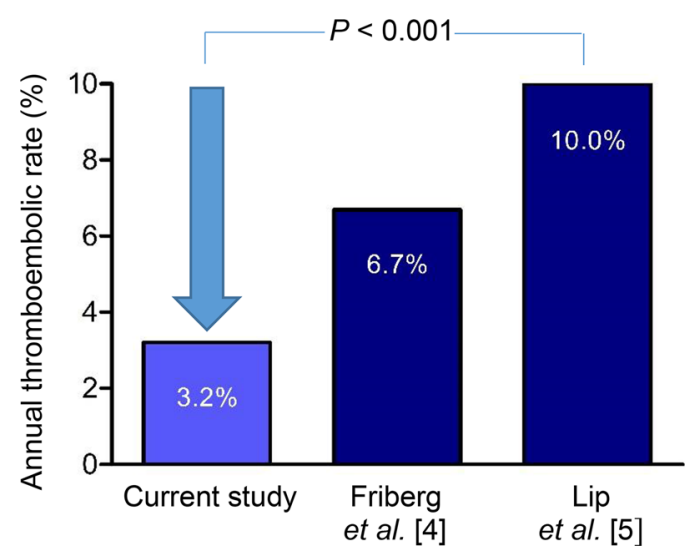

Fig. 3 Effectiveness in risk reduction of thromboembolism and bleeding. Observed annual rates of thromboembolism and bleeding events in the study population during follow-up period compared with the expected rate based
AF was similar to that of ACP, despite the fact that patients were on OAC 45 days post-implant. In all cases reported so far, short-duration OAC therapy or low molecular weight heparin were effective in resolving thrombi.

\section{Study Limitations}

This study has all the limitations of a single-center retrospective observational study. The sample size of the study was small and no inference about comparative outcomes can be made due to lack of a control group. The annual stroke rate of our population was compared with the estimated events based on the $\mathrm{CHA}_{2} \mathrm{DS}_{2}$-VASc score. Because the expected events are based on historic controls and not validated in the current study population, the possibility of a type I error cannot be ruled out. For the above reasons, randomized controlled trials are needed for further validation of the results. It must be pointed out that patients of our study were on DAPT for 6 months after the procedure. DAPT has been reported to reduce

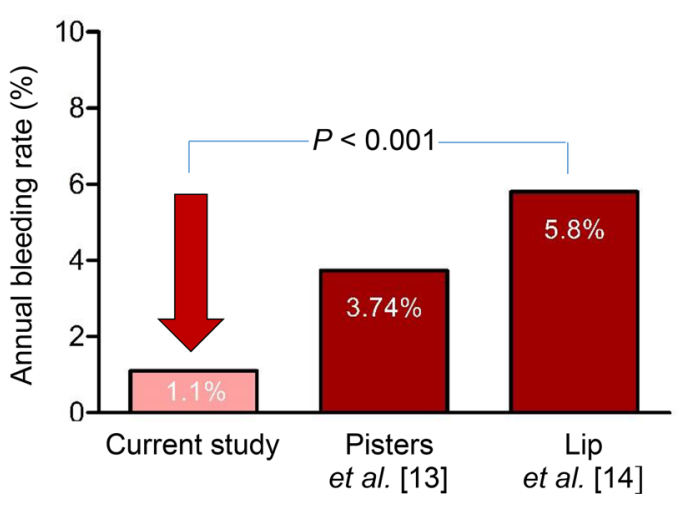

on $\mathrm{CHA}_{2} \mathrm{DS}_{2}$-Vasc and HAS-BLED score. Arrows indicate risk reductions of events compared to expected event rates obtained from earlier studies 
stroke risk. This was not considered when comparing expected and observed stroke rates, and hence an inflated benefit cannot be ruled out. Finally, echocardiographic follow-up was incomplete and events such as minimal leaks or device thrombosis may have been missed.

\section{CONCLUSIONS}

LAAC with the ACP appears to be effective with acceptable rates of periprocedural complications and low rates of thromboembolic and bleeding events at follow-up in a cohort of very high stroke and bleeding risk patients with AF. With growing experience, this new technology of LAAC using ACP may become a valid alternative for high-risk patients with AF with contraindication for OAC.

\section{ACKNOWLEDGMENTS}

No funding or sponsorship was received for this study or publication of this article. All named authors meet the International Committee of Medical Journal Editors (ICMJE) criteria for authorship for this manuscript, take responsibility for the integrity of the work as a whole, and have given final approval for the version to be published.

Disclosures. M. Abdel-Wahab has received institutional research grants from St. Jude Medical and Biotronik, is a proctor for Boston Scientifc. G. Richardt has received institutional research Grants from St. Jude Medical and Biotronik, and receives lecture fees from Edwards Lifesciences and Boston Scientific. J. Jose is currently supported by an EAPCI Grant in Interventional Cardiology, which is partially sponsored by Medtronic. Julia Kebernik, Björn Stöcker, and Volker Geist have no conflicts of interest to disclose.
Compliance with Ethics Guidelines. All procedures followed were in accordance with ethical standards of the responsible committee on human experimentation (institutional and national) and with the Helsinki Declaration of 1964, as revised in 2013. Informed written consent for the procedure was obtained from all patients.

Open Access. This article is distributed under the terms of the Creative Commons Attribution-NonCommercial 4.0 International License (http://creativecommons.org/licenses/ by-nc/4.0/), which permits any noncommercial use, distribution, and reproduction in any medium, provided you give appropriate credit to the original author(s) and the source, provide a link to the Creative Commons license, and indicate if changes were made.

\section{REFERENCES}

1. Conti CR. Atrial fibrillation, transesophageal echo, electrical cardioversion, and anticoagulation. Clin Cardiol. 1994;17:639-40.

2. Hart RG, Pearce LA, Aguilar MI. Meta-analysis: antithrombotic therapy to prevent stroke in patients who have nonvalvular atrial fibrillation. Ann Intern Med. 2007;146:857-67.

3. Mant J, Hobbs FD, Fletcher K, et al. Warfarin versus aspirin for stroke prevention in an elderly community population with atrial fibrillation (the Birmingham Atrial Fibrillation Treatment of the Aged Study, BAFTA): a randomised controlled trial. Lancet. 2007;370:493-503.

4. Friberg L, Rosenqvist M, Lip GY. Evaluation of risk stratification schemes for ischaemic stroke and bleeding in 182678 patients with atrial fibrillation: the Swedish Atrial Fibrillation cohort study. Eur Heart J. 2012;33:1500-10.

5. Lip GY, Frison L, Halperin JL, et al. Identifying patients at high risk for stroke despite anticoagulation: a comparison of contemporary stroke risk stratification schemes in an anticoagulated atrial fibrillation cohort. Stroke. 2010;41:2731-8. 
6. Blackshear JL, Odell JA. Appendage obliteration to reduce stroke in cardiac surgical patients with atrial fibrillation. Ann Thorac Surg. 1996;61:755-9.

7. Holmes DR Jr, Doshi SK, Kar S, et al. Left atrial appendage closure as an alternative to warfarin for stroke prevention in atrial fibrillation: a patient-level meta-analysis. J Am Coll Cardiol. 2015;65:2614-23.

8. Kefer J, Vermeersch P, Budts W, et al. Transcatheter left atrial appendage closure for stroke prevention in atrial fibrillation with Amplatzer cardiac plug: the Belgian Registry. Acta Cardiol. 2013;68:551-8.

9. Urena $M$, Rodés-Cabau J, Freixa $X$, et al. Percutaneous left atrial appendage closure with the amplatzer and contraindications to anticoagulation therapy cardiac plug device in patients with nonvalvular atrial fibrillation. J Am Coll Cardiol. 2013;62:96-102.

10. Santoro G, Meucci F, Stolcova $M$, et al. Percutaneous left atrial appendage occlusion in patients with non-valvular atrial fibrillation: implantation and up to four years follow-up of the AMPLATZER Cardiac Plug. EuroIntervention. 2014. doi:10.4244/EIJY14M10_13.

11. Tzikas A, Shakir S, Gafoor S, et al. Left atrial appendage occlusion for stroke prevention in atrial fibrillation: multicentre experience with the AMPLATZER Cardiac Plug. EuroIntervention. 2015;10(10). doi:10.4244/EIJY15M01_06.

12. Mehran R, Rao SV, Bhatt DL, et al. Standardized bleeding definitions for cardiovascular clinical trials. A consensus report from the bleeding academic research consortium. Circulation. 2011;123:2736-47.

13. Pisters R, Lane DA, Nieuwlaat R, et al. A novel user-friendly score (HAS-BLED) to assess 1-year risk of major bleeding in patients with atrial fibrillation: the Euro Heart Survey. Chest. 2010;138:1093-100.

14. Lip GY, Frison L, Halperin JL, et al. Comperative validation of a novel risk score for predicting bleeding risk in anticoagulated patients with atrial fibrillation: the HAS-BLED (Hypertension, Abnormal Renal/Liver Function, Stroke, Bleeding History or Predisposition, Labile INR, Elderly, Drugs/Alcohol Concomitantly) score. J Am Coll Cardiol. 2011;57:173-80.

15. Connoly SJ, Ezekowitz MD, Yusuf S, et al. Dabigatran versus warfarin in patients with atrial fibrillation. N Engl J Med. 2009;361:1139-51.
16. Patel MR, Mahaffey KW, Garg J, et al. Rivaroxaban versus warfarin in nonvalvular atrial fibrillation. N Engl J Med. 2011;365:883-91.

17. Granger CB, Alexander JH, McMurray JJ, et al. Apixaban versus warfarin in patients with atrial fibrillation. N Engl J Med. 2011;365:981-92.

18. Pollack CV Jr, Reilly PA, Eikelboom J, et al. Idarucizumab for dabigatran reversal. $\mathrm{N}$ Engl J Med. 2015;373:511-20.

19. Block PC, Burstein S, Casale PN, et al. Percutaneous left atrial appendage occlusion for patients in atrial fibrillation suboptimal for warfarin therapy: 5-year results of the PLAATO (Percuaneous Left Atrial Appendage Transcatheter Occlusion) Study. JACC Cardiovasc Interv. 2009;2:594-600.

20. Reddy VY, Doshi SK, Sievert H, et al. Percutaneous left atrial appendage closure for stroke prophylaxis in patients with atrial fibrillation: 2.3-Year Follow-up of the PROTECT AF (Watchman Left Atrial Appendage System for Embolic Protection in Patients with Atrial Fibrillation) Trial. Circulation. 2013;127:720-9.

21. Holmes DR Jr, Kar S, Price MJ, et al. Prospective randomized evaluation of the Watchman Left Atrial Appendage Closure device in patients with atrial fibrillation versus long-term warfarin therapy: the PREVAIL trial. J Am Coll Cardiol. 2014;64:1-12.

22. Reddy VY, Mobius-Winkler S, Miller MA, et al. Left atrial appendage closure with the Watchman device in patients with a contraindication for oral anticoagulation: the ASAP study (ASA Plavix Feasibility Study With Watchman Left Atrial Appendage Closure Technology). J Am Coll Cardiol. 2013;61:2551-6.

23. Bergmann MW, Landmesser U. Left atrial appendage closure for stroke prevention in non-valvular atrial fibrillation: rationale, devices in clinical development and insights into implantation techniques. EuroIntervention. 2014;10:497-504.

24. Reddy VY, Holmes D, Doshi SK, et al. Safety of percutaneous left atrial appendage closure: results from the Watchman Left Atrial Appendage System for Embolic Protection in Patients With AF (PROTECT AF) clinical trial and the Continued Access Registry. Circulation. 2011;123:417-24.

25. Aminian A, Lalmand J, Tzikas A, et al. Embolization of left atrial appendage closure devices: a systematic review of cases reported with the watchman device and the amplatzer cardiac plug. Catheter Cardiovasc Interv. 2015;86:128-35. 